\title{
Symplasmic transport and phloem loading in gymnosperm leaves
}

\author{
Johannes Liesche • Helle Juel Martens • \\ Alexander Schulz
}

Received: 21 September 2010 / Accepted: 1 November 2010 / Published online: 24 November 2010

(C) Springer-Verlag 2010

\begin{abstract}
Despite more than 130 years of research, phloem loading is far from being understood in gymnosperms. In part this is due to the special architecture of their leaves. They differ from angiosperm leaves among others by having a transfusion tissue between bundle sheath and the axial vascular elements. This article reviews the somewhat inaccessible and/or neglected literature and identifies the key points for pre-phloem transport and loading of photoassimilates. The pre-phloem pathway of assimilates is structurally characterized by a high number of plasmodesmata between all cell types starting in the mesophyll and continuing via bundle sheath, transfusion parenchyma, Strasburger cells up to the sieve elements. Occurrence of median cavities and branching indicates that primary plasmodesmata get secondarily modified and multiplied during expansion growth. Only functional tests can elucidate whether this symplasmic pathway is indeed continuous for assimilates, and if phloem loading in gymnosperms is comparable with the symplasmic loading mode in many angiosperm trees. In contrast to angiosperms, the bundle sheath has properties of an endodermis and is equipped with Casparian strips or other wall modifications that form a domain border for any apoplasmic transport. It constitutes a key point of control for nutrient transport, where the opposing flow of mineral nutrients and photoassimilates has to be accommodated in each single cell, bringing to mind the principle of a revolving door. The review lists a
\end{abstract}

Handling Editor: Manfred Heinlein

J. Liesche $\cdot$ H. J. Martens $\cdot$ A. Schulz $(\bowtie)$

Department of Plant Biology and Biotechnology, University of Copenhagen,

Thorvaldsensvej 40,

DK-1871, Frederiksberg C, Denmark

e-mail: als@life.ku.dk number of experiments needed to elucidate the mode of phloem loading in gymnosperms.

Keywords Bundle sheath · Endodermis · Plasmodesmata . Transfusion tissue $\cdot$ Strasburger cells $\cdot$ Xylem

\section{Introduction}

In angiosperms, the regulation of phloem loading is usually attributed to the companion cells of minor veins. According to textbooks, transport sugars are actively concentrated in the sieve element-companion cell complexes, leading to an osmotically generated pressure flow from source to sink organs. As active loading strategies, two modes of phloem loading are well characterized: apoplasmic loading, depending on plasma membrane transporters, and symplasmic loading, depending on synthesis of sugar polymers and abundance of plasmodesmata between companion cells and bundle sheath, respectively. The role of cells of the prephloem pathway between mesophyll and bundle sheath is easily overlooked. This is evident for the passive symplasmic loading strategy, where the highest sugar concentrations are found in the mesophyll rather than the phloem (see Davidson et al. 2010, this issue). Moreover, only few studies have considered the intersection where the prephloem transport of photoassimilates is interwoven with the post-xylem transport of water, so that opposite streams might have influence on each other.

For gymnosperms, the mode of phloem loading has not been assessed yet, neither is it known whether both apoplast and symplasm are involved in it. Gymnosperm sieve elements are not associated with companion cells, but with Strasburger cells that are ontogenetically unrelated. However, their ultrastructure and the pore-plasmodesmata 
contacts are similar to companion cells (Schulz 1990). Whether and how they might contribute to phloem loading is unknown. The vascular strands in the leaves of all gymnosperms are separated from the mesophyll by a particular tissue, the transfusion tissue, and by an endodermis-like bundle sheath. All these special features indicate that assimilate loading might involve mechanisms and pathways hitherto not found in angiosperms.

Most angiosperm plant genera could be assigned to one of three loading types (Rennie and Turgeon 2009). In some cases even species-specific characteristics have been identified (Zhang and Turgeon 2009; Gil 2010). As a general rule for sucrose transporting angiosperms, active apoplasmic loading, i.e., sugar uptake into symplasmically isolated sieve element-companion cell complexes, appears to be predominant in herbaceous plants, while passive symplasmic loading, i.e., sugar diffusion from the mesophyll into sieve element-companion cell complexes phloem that are well-connected to the bundle sheath by plasmodesmata, is most common in angiosperm trees (Turgeon 2010, see also Turgeon and Medville 2010 this issue). All gymnosperms seem to translocate sucrose in the phloem, even though this has not been studied systematically (Shiroya et al. 1962; Willenbrink and Kollmann 1966; Willenbrink 2002).

Understanding the mechanisms of phloem loading in gymnosperm trees will cast light on the evolution and significance of long distance transport mechanisms in land plants, just in the context of recent discussions of passive phloem loading in angiosperm trees (Turgeon 2010). Gymnosperm leaves have fascinated scientists already many years back. Münch, who postulated the mechanism of pressure-driven phloem transport in 1930, was attentive for the peculiarities of gymnosperm needles and the role of the transfusion tissue between bundle sheath and vascular tissue (Fig. 1a). Since older references are often somewhat inaccessible, we chose to compile data and reprint some selected electron micrographs from the relevant literature. Most papers available covered single aspects of the structure and physiology of gymnosperm leaves only. The aim of this review is to give an integrated overview of their transport pathways, highlight significant control key points and identify open questions that have to be studied in order to understand phloem loading in gymnosperms.

\section{Leaf morphology and anatomy}

Taxonomically, gymnosperms are divided in conifers, cycads, Gnetophytes with Gnetum, Welwitschia, and Ephe$d r a$, and the Ginkgophytes with the only representative Ginkgo biloba. They are mainly evergreen trees, shrubs and a few lianas and well adapted to extreme habitats such as boreal forests with long winter periods, but in some cases also to tropical or desert environments. Most studies on gymnosperms focus on Pinaceae, by far the most abundant and economically important taxon of gymnosperms. Most Pinaceae have needle-shaped leaves, but gymnosperms in general have developed many different leaf shapes such as scales, pinnate, and broad leaves. The xeromorphic character of all gymnosperm leaves is reflected by an epidermis with thick cutinized outer walls that is often reinforced by hypodermal sclerenchyma fibers. Stomata are usually arranged in rows on the adaxial side and are sunken into the leaf surface. In most conifers, a variable number of resin ducts can be found in the needle periphery. In needles, vascular bundles are arranged in one or two central strands. However, Ginkgo leaf blades show a dichotomous venation pattern, Welwitschia parallel venation with anastomoses and Gnetum a reticulate venation with at least five vein classes, similar to most dicots.

Independent of shape, the vascular tissue of gymnosperm leaves consists of axial xylem and phloem elements embedded in the characteristic transfusion tissue. This tissue is again bordered by a conspicuous bundle sheath which for a number of gymnosperms was described to have similarities to a root endodermis. The transfusion tissue is composed of transfusion tracheids and transfusion parenchyma cells that are in contact with the axial xylem elements (tracheids) and Strasburger cells of the phloem, respectively (Fig. 1). Structure, amount, and arrangement of the transfusion tissue vary in the different species (Esau 1965).

Sieve elements belong to the axial phloem system and are linked by sieve areas in their long-tapering end walls. When mature, their cytoplasm is reduced to plastids, mitochondria and ER, as is that of angiosperm sieve elements (Schulz 1990). In stark contrast to the sieve pores of angiosperms, tubular ER is a regular compound in the sieve pores of gymnosperms and forms complexes on either side of the sieve areas, as visualized by confocal microscopy in live phloem (Schulz 1992). Unlike the angiosperm companion cells, Strasburger cells are ontogenetically unrelated to sieve elements and only partially in contact with them. Where they are, they develop extensive poreplasmodesmata contacts, covered by complexes of tubular ER on the sieve element side (Schulz 1990; Glockmann and Kollmann 1996). In the collection phloem of needles, Strasburger cells are typically flanking the axial sieve elements in two to four layers, and may in addition occur interspersed in the axial phloem (Fig. 1a). Gnetum leaves have minor veins with only one tracheid and one to two sieve elements in the smallest vein class. Here, the Strasburger cells are larger in diameter than the sieve elements (Fig. 1b). Besides transfusion tissue and vascular elements, there may occur sclerenchyma fibers and parenchyma cells in the vascular bundles (Fig. 1a). 


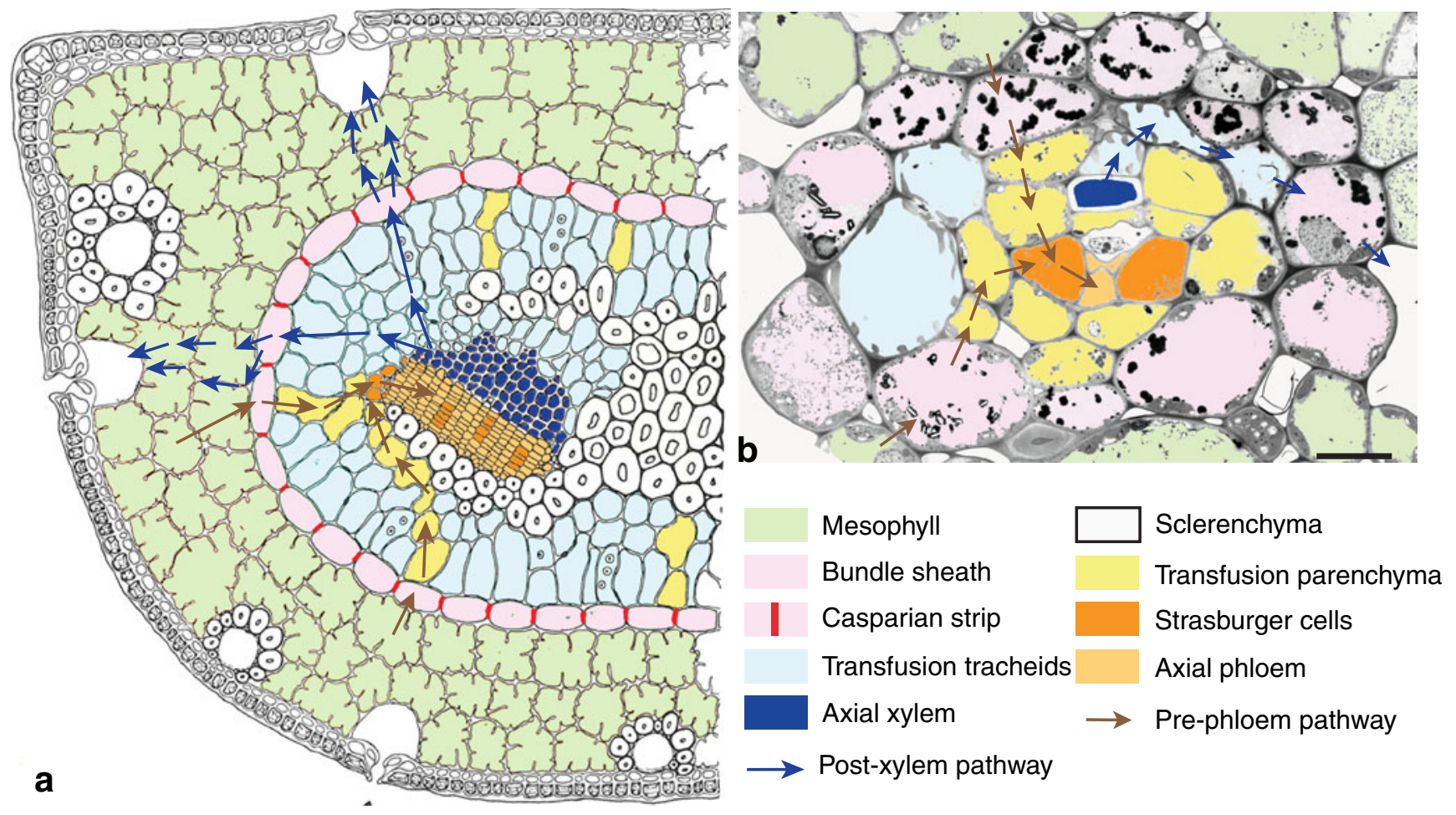

Fig. 1 Illustration of the different cell types and transport pathways in gymnosperm leaves on a schematic drawing of a $P$. sylvestris needle cross section (a) and an electron micrograph of a Gnetum gnemon fifth class minor vein (b). The pre-phloem pathway (brown arrows) from the mesophyll is symplasmic and crosses bundle sheath, transfusions parenchyma and Strasburger cells before approaching the sieve elements. The post-xylem pathway (blue arrows) in pines starts in

The transfusion tracheids are dead cells and a part of the transfusion tissue surrounding the axial vascular elements. They are characteristic for all leaves of gymnosperms (Fig. 1, de Bary 1877; Strasburger 1891). Transfusion tracheids are not only found in needle-bearing gymnosperms with one or two central veins, but also in the smallest veins of Gnetum, which has more than five vein classes (Fig. 1b). Gambles and Dengler (1982) identified four distinct groups of transfusion tracheids in Pinus resinosa based on their anatomy. Different degree of wall modification and pit morphology suggest that some transfusion tracheids are optimized for water transport whereas others are more important for water storage and support. The lignified walls might enable them to maintain their shape under great water stress as an adaptation to arid conditions (Gadek and Quinn 1988). The role in water conductance, first proposed by Worsdell (1897) was demonstrated with fluorescent tracers in the transpiration stream (Heimerdinger 1951). In addition to increasing drought resistance, the capacity to store water in the transfusion tissue might serve as prevention of frost damage to living cells (Roden et al. 2009).

The transfusion parenchyma forms bridges of living cells between the outer Strasburger cells and the bundle transfusion tracheids and has to enter the bundle sheath via the inner tangential wall, since the radial walls are sealed by the suberized Casparian strips (red). From the bundle sheath onwards it is not known to which extent the transpiration stream follows an apoplasmic, symplasmic, or transcellular route towards the sub-stomatal chambers (indicated here by the parallel arrows in a); scale bar $10 \mu \mathrm{m}$; a based on Fig. 23 in Münch (1930)

sheath (Fig. 1). The ratio of parenchyma cells to tracheids in the transfusion tissue varies between $18 \%$ and $88 \%$ in pine species (Lederer 1955). The two cell types can be understood as a physiological unit (Canny 1993).

An endodermis-like bundle sheath was described to be a consistent feature for needles of Pinaceae (Lederer 1955; Esau 1977; see Fig. 2a). Suberization and/or lignification of radial and transverse walls of the bundle sheath, including typical Casparian strips, have indeed been detected in needles of numerous Pinaceae species by microscopic staining techniques, electron microscopy and spectroscopic analysis (Fig. 2a, Carde 1978; Canny 1993; Kaku 1971; Scholz and Bauch 1973; Soda et al. 2000; Wu et al. 2001; $\mathrm{Wu}$ et al. 2003). Casparian strips have not been reported outside the Pinaceae, but representatives of other gymnosperm families have often wall modifications in the bundle sheath cells. The walls of bundle sheath cells in Cycas revoluta leaves are thickened and lignified next to the vascular tissue, and in leaves of G. biloba each of the numerous vascular bundles has a lignified endodermis (Esau 1977; Lederer 1955; see Fig. 2b). Rehydration experiments demonstrated a high radial resistance for water flow in all tested gymnosperms, which was attributed to the hydraulic isolation of vascular and photosynthetic tissue by 


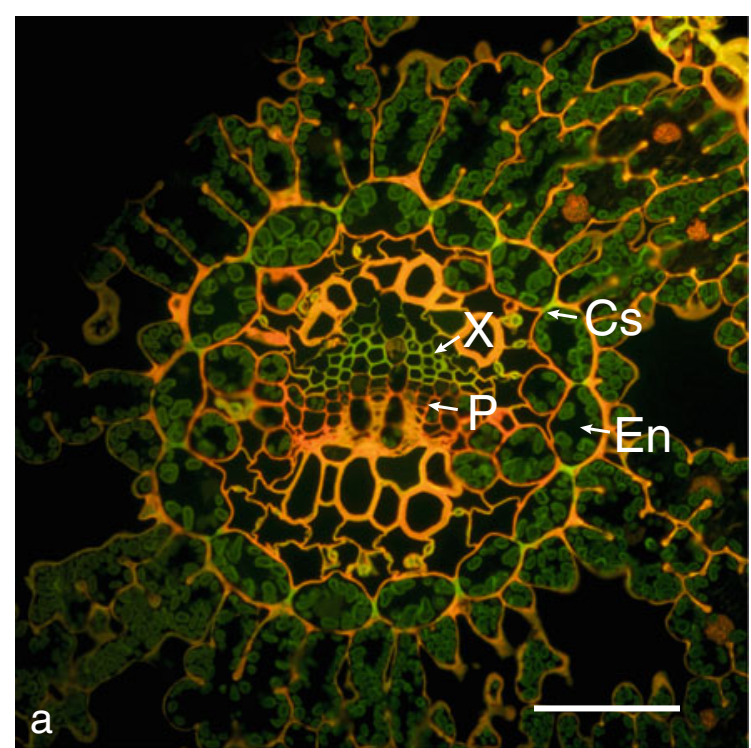

Fig. 2 Fluorescence micrographs of cross-sections of fixed leaves stained with the component-specific cell wall dyes Berberine hemisulfate (lignin and suberin), Aniline blue (quenching of nonspecific Berberine hemisulfate staining) and Coriphosphine B (pectin) to

the bundle sheath (Scholz and Bauch 1973; Zwieniecki et al. 2007). As an apoplasmic barrier for the transpiration stream the bundle sheath might play an important role in drought resistances (Soar 1922) and frost tolerance (Roden et al. 2009; Kaku 1971).

The complex anatomy of gymnosperm leaves has significant implications, both for the post-xylem and pre-phloem pathway, and thus for the transport of water, mineral nutrients and photoassimilates. The few functional studies on transport in gymnosperm leaves dealt only with water transport (Wu et al. 2005; Scholz and Bauch 1973; Zwieniecki et al. 2007) with the exception of simple tracer experiments done by Heimerdinger (1951) and Canny (1993). Apart from these two reports, the symplasmic domain has only been addressed by ultrastructural analysis of cell connections. The assimilate pathway from the mesophyll to the sieve elements in the phloem has never been looked at as a whole.

\section{The symplasm}

The symplasm of gymnosperm leaves consists of all living cells (presumably except for guard cells) and can be divided into an inner, vascular part, and an outer part starting with the bundle sheath. Inner and outer parts are isolated from each other by the extended system of transfusion tracheids except for the transfusion parenchyma bridges (see Fig. 1a). Moreover, in many gymnosperms the upper and lower side of the vasculature is additionally isolated by sclerenchyma cells (Lederer 1955). The only connection between the axial vascular phloem tissue and the bundle sheath is accordingly

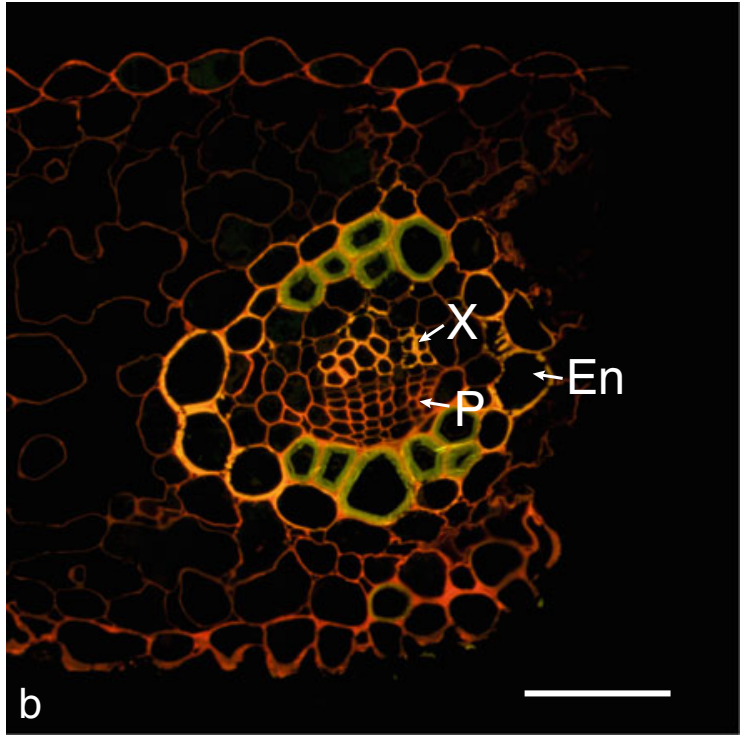

highlight wall modifications, especially the Casparian strip $(C s)$ in the Pinus parviflora needle (a) and the suberized bundle sheath of $G$. biloba (b); En endodermis-like bundle sheath, $P$ phloem, $X$ xylem; scale bars $50 \mu \mathrm{m}$

established through Strasburger cells, flanking the axial phloem, and transfusion parenchyma (Carde 1978).

The rate of symplasmic transport under physiological condition depends upon the size-exclusion limit (SEL) and frequency of plasmodesmata between the cells in question, both of which can be modulated during development (Kim and Zambryski 2005) and under stress conditions (Schulz 1995, 1999). For the study of symplasmic domain borders in gymnosperm leaves, plasmodesmal frequencies and the movement of synthetic tracers can be utilized. Interesting domain borders, such as those in the phloem, shown in transformed angiosperms to having a SEL allowing GFP passage, cannot be tested with reporter genes in gymnosperms as long as cell-specific transformation protocols are not available.

\section{Cells of the pre-phloem pathway are connected radially but not axially in pine needles}

In pine needles, all cells but the sieve elements are symplasmically isolated in axial direction, i.e., along the needle (Münch 1930; Strasburger 1891). The mesophyll cells are in most pine species organized in rows with airfilled spaces in between, again isolating them in axial direction. The endodermis cells are connected by pit fields in the radial, not in the end walls (Canny 1993; Carde 1978; Walles 1973; Fig. 3c and d). Transfusion parenchyma cells form a network through the transfusion tissue, again with preferentially radial contacts to each other (Heimerdinger 1951). 
Modeling of the hydraulic parameters in leaves led Zwieniecki et al. (2004) to conclude that development of single-veined leaves is accompanied by a high radial water resistance outside the stele during leaf expansion. This conclusion is compatible with the view that water exits from the stele primarily through the protoplasts of the endodermislike bundle sheath. The endodermis layer itself does not allow water transport in axial direction: neither through the apoplast, since the entire end wall of endodermis cells is sealed by suberin and/or lignin, nor through the symplasm, since the end walls are not crossed by pit fields/plasmodesmata (Carde 1978). Accordingly, assimilates entering the endodermis from the mesophyll, have to continue radially via the transfusion parenchyma towards the Strasburger cells. Only the sieve elements then offer an axial transport pathway. The axial isolation of the pre-phloem pathway might be important for the stabilization of concentration and pressure gradients over the length of the needle.

\section{Branched plasmodesmata are present at all interfaces of the pre-phloem pathway in gymnosperm leaves}

Leaves of a number of different gymnosperm species have been studied by electron microscopy. For the pre-phloem pathway the micrographs of plasmodesmata indicate unifying features across all gymnosperm species. We reprinted some micrographs from less accessible papers and combined them in Fig. 3, which shows that groups of plasmodesmata with more or less extensive median cavities are typically combined in fields at all interfaces from mesophyll to phloem: mesophyll-endodermis-transfusion parenchyma-Strasburger cell-sieve element (Fig. 3a-e, g; see also Parameswaran and Liese 1970; Boddi et al. 2002; Carde 1974, 1978; Gambles and Dengler 1982; Glockmann and Kollmann 1996; Harris 1972; Scholz and Bauch 1973; Walles 1973). While most plasmodesmata on the pre-phloem pathway are located in non-elevated primary pit fields, those linking Strasburger cells are located in conspicuous, dome-shaped wall thickenings (Fig. $3 g$ and Carde 1974: Fig. VIc). Staining identified the thickenings to be primary wall depositions and to consist of hemicelluloses, pectin, and callose rather than cellulose (Carde 1974; Glockmann and Kollmann 1996). Branched plasmodesmata are a regular feature in mesophyll tissue, also in angiosperms (see e.g., Kühn et al. 1996) and seem to be the result of secondary modification of primary plasmodesmata (see Faulkner and Oparka 2009). However, the formation of large dome-shaped wall thickenings containing numerous plasmodesmata is characteristic for gymnosperm leaves. Only the companion cell side of pore-plasmodesmata units between sieve element and companion cell of angiosperms is somewhat comparable, but much less complex, than that of Strasburger cells.

\section{Contacts of the transfusion parenchyma with Strasburger cells}

Carde (1973) distinguished between three types of Strasburger cells: inner ones abutting the sieve elements, the most abundant central ones, and outer ones characterized by presence of chloroplasts. The inner and central Strasburger cells can easily be identified based on their domeshaped wall thickenings, while the chloroplast-containing outer cells have regular pit fields (see Fig. 3e). Since Strasburger cells also from non-conifers are characterized by having plasmodesmal connections in dome-shaped wall thickenings (Glockmann and Schulz, unpublished) we suggest to name only those cells "Strasburger cells" in the gymnosperm leaf which have dome-shaped wall thickening, and to describe the other living parenchyma cells inside the bundle sheath (with or without chloroplasts) as transfusion parenchyma (see also Fig. 1). Carde himself called the entire system of Strasburger cells flanking the axial phloem transfer tissue ("Le tissu de transfert", Carde 1973) and assumed that the Strasburger cells play an important role in the transfer of assimilates to the phloem.

\section{Development of dome-shaped symplasmic contacts in Strasburger cells}

A consistent feature of Strasburger cells in all gymnosperms are the dome-shaped symplasmic contacts towards other Strasburger cells and towards sieve elements (Carde 1974; Gambles and Dengler 1982; Parameswaran and Liese 1970). A detailed ultrastructural study on the development of these contacts in Metasequoia glyptostroboides by Glockmann and Kollmann (1996) indicated that these contacts develop from primary plasmodesmata in the thin walls between young Strasburger cells (Fig. 3f). During differentiation and expansion growth of the needle, the wall becomes thicker and the primary plasmodesmata secondarily modified by multiple branching (Fig. 3g, i; see Glockmann and Kollmann 1996). The only remnant of the primary plasmodesmata is then the median cavity which dilates through sheering forces, introduced by expansion growth of the needle (Fig. 3i).

Branching might be seen as a means to maintain a high degree of symplasmic contact between the Strasburger cells. Interestingly, the plasmodesmal orifices have the typical neck region (Fig. 3h), indicative for the need to control plasmodesmal transport (Glockmann and Kollmann 1996). Callose and/or protein interactions were discussed to shape the plasmodesmal neck region, which can be dilated under physiological conditions (Schulz 1995, 1999; Lucas et al. 2009). The elaborate and tubular ER system in the dome-shaped wall thickenings might on one hand be a 

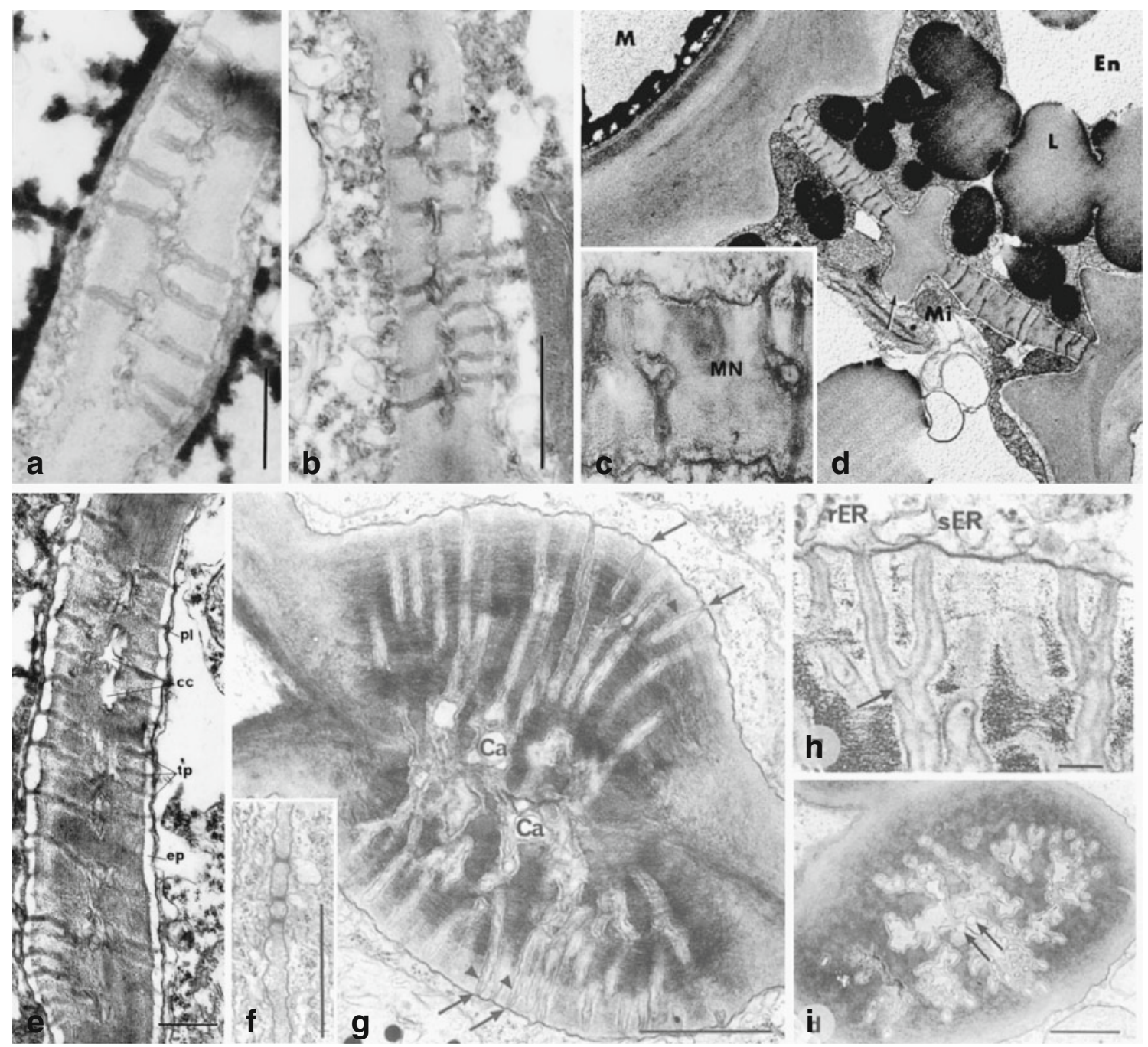

Fig. 3 Electron micrographs of plasmodesmata at the interfaces between two mesophyll cells (a, Boddi et al. 2002), a mesophyll (left) and an endodermis cell (right) (b, Boddi et al. 2002), two endodermis cells (radial wall) (c, Carde 1978; d, Gambles and Dengler 1982), a transfusion parenchyma cell (left) and a Strasburger cell (right) (e, Carde 1974), and two Strasburger cells (f-i, Glockmann and Kollmann 1996). All contacts in mature cell walls are characterized by branched plasmodesmata and more or less extended median cavities (all but f). Simple plasmodesmata connect young Strasburger cells (f), but get secondarily modified in mature Strasburger cells by

consequence of the way the original plasmodesmata get secondarily modified by multiplication of plasmodesmal arms, selective deposition of new wall material and local loosening of the existing wall. This is supported by the different wall composition of the dome-shaped wall regions (Carde 1974; Glockmann and Kollmann 1996; see also discussion in Ehlers and Kollmann 1996, 2001; Faulkner et al. 2008; Faulkner and Oparka 2009; Burch-Smith et al. 2010, this issue). On the other hand, the elaborate ER system of Strasburger cell plasmodesmata might indicate an involvement of the ER in phloem loading (Carde 1974; Glockmann and Kollmann 1996). The presence of ER the development of dome-shaped wall thickenings that contain complex branched plasmodesmal connections (g)-(i). Neck region at the orifices of these contacts (h). Arrow in $\mathbf{d}=$ thickened wall in the center of the pit, in $\mathbf{g}=$ neck regions, in $\mathbf{h}=$ branching site, in $\mathbf{i}=$ bulblike dilation in cavity, arrowheads in $\mathbf{g}=$ expanded sleeve regions, $\mathrm{Ca}$ and $c c$ central cavity, $E n$ endodermal cell, ep periplasmatic space, $L$ lipid droplet, $M$ mesophyll cell, $M i$ mitochondria, $M N$ median nodule, $p l$ plasmalemma, $r E R$ rough endoplasmatic reticulum, $s E R$ smooth endoplasmatic reticulum, tp desmotuble; scale bars $1 \mu \mathrm{m}(\mathbf{a}, \mathbf{b}, \mathbf{g}, \mathbf{f}, \mathbf{i})$, $5 \mu \mathrm{m}(\mathbf{e}), 0.1 \mu \mathrm{m}(\mathbf{h})$

complexes on both sides of live sieve areas inspired Schulz $(1992,2005)$ to discuss that the ER has an active function in phloem transport of gymnosperms.

\section{Continuity of the pre-phloem pathway with the sieve elements indicates a symplasmic mode of phloem loading}

According to the cited electron microscopic papers, there is no indication for an isolation of the phloem from the prephloem pathway, otherwise characteristic for the closed 
minor vein configuration of apoplasmic phloem loaders (Gamalei 1989). On the contrary, the pre-phloem pathway of gymnosperms seems to provide a well-connected symplasmic pathway continuous all the way to the conducting sieve elements. The connections between the inner Strasburger cells and the sieve elements are poreplasmodesmata contacts with many branched plasmodesmata within the wall thickenings of the Strasburger cell side (Carde 1974; Gambles and Dengler 1982; Glockmann and Kollmann 1996; Parameswaran and Liese 1970) and identical to the ones found in primary and secondary stem phloem of conifers (see Schulz 1990).

Continuity of symplasmic connections would suggest that sucrose produced in the mesophyll cells reaches the phloem by diffusion, similar to what was proposed for many angiosperm tree species (Turgeon et al. 2001; Turgeon 2010). If so, "phloem loading" happens already on the pre-phloem pathway in gymnosperms (see Turgeon and Medville 1998). Electron microscopy and localization of plasmodesmal targeted proteins can, however, not tell whether plasmodesmata are functional. For example, even though it is generally agreed that functional guard cells are symplasmically isolated, reporter protein experiments and random electron micrographs might falsely indicate presence of plasmodesmata (Itaya et al. 1998: Fig. 5c; Pallas and Mollenhauer 1972). Evidence for truncation of plasmodesmata towards guard cells was given by serial sectioning (Wille and Lucas 1984).

An indication that the plasmodesmata in the pre-phloem pathway of gymnosperms are indeed functional was provided by early experiments with fluorescent tracers. The cytoplasmic tracer fluorescein, applied to a cut surface on the lower side of a Pinus sylvestris needle, reached the endodermis, transfusion parenchyma and the phloem after short incubation (Heimerdinger 1951). Whether the tracer approached the sieve elements is not clear, since Heimerdinger did not discriminate between the cell types of the phloem. Movement of fluorescein is indicative for symplasmic continuity, but cannot detect restrictions in symplasmic domains such as those resulting from small SEL and/or low plasmodesmal frequencies.

\section{Opposite transpiration and assimilate streams demand tight control of water, ion and sucrose transport across the plasma membranes of bundle sheath and transfusion parenchyma}

A symplasmic mode of phloem loading, as indicated by the presence of plasmodesmata all along the pre-phloem pathway, raises questions based on the unique anatomy of gymnosperm leaves. It is dramatically different from that of angiosperms in two aspects: (1) the particular features of the bundle sheath and (2) the transfusion tissue between bundle sheath and axial vascular elements.

1. The endodermis-like bundle sheath in pines is the bottleneck for outward transport of water and mineral nutrients (Fig. 1: blue arrows). Since the radial walls block apoplasmic transport efficiently (Canny 1993), the plasma membrane of each bundle sheath cell has to contain a sufficient number of aquaporins (Voicu et al. 2009) and other transporter proteins to take up for water and ions from the transfusion tracheids. Also in non-pine gymnosperms does the bundle sheath seem to be a barrier for water transport, even though they do not have an obvious Casparian strip. This was established by rehydration studies of Metasequoia, Ginkgo, and Gnetum leaves (Zwieniecki et al. 2007). The flow of the transpiration stream out of the bundle sheath towards stomata could be apoplasmic, symplasmic or transcellular. This means that water could re-enter the apoplast and stay there, move through the plasmodesmata to the neighboring mesophyll cell or get to the mesophyll by crossing the plasma membranes of both, bundle sheath cell and mesophyll cell (see blue arrows in Fig. 1). This situation reflects the general discussion on post-xylem movement of water where a conclusion has yet to be reached (Sack and Holbrook 2006).

Simultaneous to the outward flow of water, all the inward transport of assimilates from the mesophyll to the transfusion parenchyma has to pass the bundle sheath (see Fig. 1: brown arrows). Already, Münch (1930) doubted that the opposing flow of water and photoassimilates could be accommodated in the same cells. Once inside the cytosol of the bundle sheath, an outward bulk flow of water would meet the inward diffusion of photoassimilates resulting in a very inefficient assimilate transport. The situation appears even less conceivable in case that the plasmodesmata in the outer bundle sheath wall are involved in water transport. This would imply that water and assimilates had to move opposite to each other in their cytoplasmic sleeves.

2. The thin network of transfusion parenchyma is the only symplasmic contact between the bundle sheath and the phloem for the inward assimilate transport. The transfusion parenchyma cells have a large surface area towards dead transfusion tracheids that are filled with water and mineral nutrients. The content of solutes in the apoplast is generally underestimated, and the concentrations of solutes in the xylem-lumen apoplast are different from those in the cell wall apoplast (Canny 1995). A steep gradient in water potential across the plasma membrane of the transfusion parenchyma cells would certainly lead to a considerable leakage of sucrose. Indeed, synthetic apoplasmic tracers, fed 
through the xylem, end up at the plasma membrane of the transfusion parenchyma and even cross this membrane after long chase periods (Canny 1993). As shown by autoradiography, radiolabelled aspartate was collected by the transfusion parenchyma and returned quickly via Strasburger cells to the phloem. Some of the label appeared also in the mesophyll. This led Canny (1993) to conclude that active transporters in the transfusion parenchyma recover solutes from the transpiration stream. How much sucrose on its way towards the Strasburger cells might leak into the apoplast of the transfusion tracheids, to be retrieved by membrane transporter activity, has yet to be determined.

As a solution to the challenges listed above, an elegant scenario would be the compartmentalization of water and assimilate transport in bundle sheath and transfusion parenchyma. In the context of this review it might be speculated that water and mineral nutrients could already be taken up by the transfusion parenchyma and access the cytosol en-route to the bundle sheath, as indicated by Canny's tracer experiments. Obviously, the used fluorochrome sulforhodamine $\mathrm{G}$ is an imperfect apoplasmic tracer that over time can cross the plasma membrane (Canny 1993). Sucrose coming from the mesophyll could be taken up at the bundle sheath by the endoplasmic reticulum, or at any point on the way in the symplasm between bundle sheath and Strasburger cells. A special role of intercellular ER in phloem loading of conifers was already discussed by Carde (1974), Glockmann and Kollmann (1996), and Schulz (2005). This role would demand sucrose transporters for sucrose uptake into the ER at the latest in the Strasburger cells, and for efflux of sucrose into the sieve element.

Whether or not sucrose is taken up by the ER at one step of the pre-phloem pathway, a problem of opposing flows of water and assimilates in bundle sheath cells and their plasmodesmata might actually not exist, since gymnosperms have xeromorphic leaves. Transpiration might be limited to the extent that outward water transport in the bundle sheath might be diffusion rather than bulk flow. Water release across the outer plasma membrane should be tightly controlled by aquaporins and, if plasmodesmata are contributing, by their SEL. The high abundance of plasmodesmata is compatible with the assumption that they have a small SEL. Narrow plasmodesmata will inhibit bulk flow of water more than diffusion. Accordingly, if transport in and out of the bundle sheath is dominated by diffusion, there is no need for compartmentalization of water and assimilates: each molecular species would diffuse on its own in the same channels with a net movement of water outwards, and a net movement of assimilate inwards.
Feasibility of either compartmentalization or diffusional flow can only be tested by functional studies of the apoplasmic and symplasmic pathways in gymnosperm leaves and by the cloning, characterization and localization of sucrose transporters possibly involved in assimilate uptake.

\section{Conclusions and open questions}

Continuity of symplasmic contacts all the way from mesophyll to sieve elements indicate a symplasmic mode of phloem loading in gymnosperms. The endodermis-like bundle sheath seems to play a key role in control of mineral nutrient and assimilate transport, i.e., the post-xylem and the pre-phloem transport. Here, the outward mineral nutrient transport, driven by transpiration, meets the inward sugar transport, presumably driven by diffusion. Logistically, it would be the easiest way to separate these two streams by compartmentalization, where assimilates and water are physically separated. Indeed, the pathways are clearly separated in the vascular tissue inside the bundle sheath, where they are limited to the transfusion tracheids and transfusion parenchyma, respectively. Here, the pathways are kept separated by the plasma membrane of the transfusion parenchyma which controls any exchange of metabolites between apoplasmic and symplasmic pathway.

For the bundle sheath, it is conceivable that the incoming assimilates are compartmentalized in the endomembrane system, perhaps even including the vacuoles. Intercellular continuity of vacuoles through the plasmodesmal ER was shown for trichomes by Lazzaro and Thomson (1996). Scholz and Bauch reported the highest osmotic potential of the needle to occur in the endodermis-like bundle sheath and made it responsible for the exudation of water from detached needles (Scholz and Bauch 1973; see also Carde 1978). Without pointing to the compartment in question, this suggests that sugars accumulate in the bundle sheath. Where sucrose would leave the endomembrane system on its inward transport is one of the questions, only further functional experiments can solve.

The mechanism of phloem loading and transport is an enigma in gymnosperms (Schulz 1992, 2005; Glockmann and Schulz 1999). All gymnosperms studied so far have the same functional anatomy, and, thus, can be expected to have a similar phloem loading mechanism, independent of whether they have needles, scales or broad leaves.

Open questions derived from the present review are

1. Are there sucrose transporters present in gymnosperms and, if so, where are they localized?

2. Are assimilates compartmentalized in the bundle sheath? 
3. What is the role of the extended ER in the plasmodesmata traversing the dome-shaped symplasmic contacts of Strasburger cells and sieve elements?

4. Is phloem loading active or passive, and does it follow an entirely cytosolic route (through the plasmodesmal sleeves), or are there a membrane step and the desmotubular pathway involved?

In order to solve these questions, it is proposed to clone putative sucrose transporters from gymnosperms, to characterize them and to try to localize them in collection tissue and transport phloem. According to the ultrastructural data, it can be assumed that pre-phloem transport relies on a diffusion gradient from mesophyll to Strasburger cells. If this is the case, any domain borders and obstacles in this pathway have to be identified. For the cytosolic pathway fluorescence redistribution after photobleaching (FRAP) and photoactivation experiments can be utilized to quantify the transport rate between selected interfaces (Pina et al. 2009). Preliminary experiments indicate that such tracer studies are able to discriminate symplasmic and apoplasmic phloem loaders (Liesche, Martens, and Schulz, unpublished). For the identification of a putative vacuolar pathway, FRAP experiments could be undertaken using a fluorescent vacuolar marker. Autoradiography of leaves after feeding of radiolabelled with $\mathrm{CO}_{2}$ could indicate any active phloem-loading steps (Turgeon and Medville 1998; Rennie and Turgeon 2009). Finally, the sugar concentration in the cytosol and the vacuoles of the pre-phloem pathway shall be assessed in order to identify those interfaces, where active transport might contribute to pre-phloem transport and phloem loading.

Acknowledgments We thank Line Svensson Kirk and Astrid S. Andersen for the light microscopic preparations of Ginkgo and Pinus parviflora, respectively (Fig. 2), and Christl Glockmann, University of Kiel, for electron microscopic preparation and micrographs of Gnetum gnemon (Fig. 1b). All comments and suggestions from the anonymous reviewers which led to the improvement of the manuscript are gratefully acknowledged.

Conflict of interest The authors declare that they have no conflict of interest.

\section{References}

Boddi S, Bonzi LM, Calamassi R (2002) Structure and ultrastructure of Pinus halepensis primary needles. Flora 197(1):10-23

Burch-Smith TM, Stonebloom S, Xu M, Zambryski PC (2010) Plasmodesmata during development: re-examination of the importance of primary, secondary, and branched plasmodesmata structure versus function. Protoplasma
Canny MJ (1993) Transfusion tissue of pine needles as a site of retrieval of solutes from the transpiration stream. New Phytol 123 (2):227-232

Canny MJ (1995) Apoplastic water and solute movement: new rules for an old space. Annu Rev Plant Physiol Plant Mol Biol 46:215236

Carde JP (1973) Le tissu de transfert (=cellules de Strasburger) dans les aiguilles du pin maritime (Pinus pinaster ait.), I. Étude histologique et infrastructurale du tissu adulte. J Microsc 17:65-88

Carde JP (1974) Le tissu de transfert (=cellules de Strasburger) dans les aiguilles du pin maritime (Pinus pinaster Ait.), II. Caractères cytochimiques et infrastructuraux de la paroi et des plasmodesmes. J Microsc 20:51-72

Carde JP (1978) Ultrastructural studies of Pinus pinaster needlesendodermis. Am J Bot 65(10):1041-1054

Davidson A, Keller F, Turgeon R (2010) Phloem loading, plant growth form, and climate. Protoplasma

de Bary A (1877) Vergleichende Anatomie der Vegetationsorgane der Phanerogamen und Farne. Wilhelm Engelmann, Leipzig

Ehlers K, Kollmann R (1996) Formation of branched plasmodesmata in regenerating Solanum nigrum-protoplasts. Planta 199(1):126138

Ehlers K, Kollmann R (2001) Primary and secondary plasmodesmata: structure, origin, and functioning. Protoplasma 216(1):1-30. doi:10.1007/bf02680127

Esau K (1965) Plant anatomy, 2nd edn. Wiley, New York, 767pp

Esau K (1977) Anatomy of seed plants, 2nd edn. Wiley, New York, $550 \mathrm{pp}$

Faulkner C, Akman OE, Bell K, Jeffree C, Oparka K (2008) Peeking into pit fields: a multiple twinning model of secondary plasmodesmata formation in tobacco. Plant Cell 20(6):1504 1518. doi: $10.1105 /$ tpc. 107.056903

Faulkner C, Oparka K (2009) Plasmodesmata. Encyclopedia of life sciences (ELS). Wiley, Chichester

Gadek PA, Quinn CJ (1988) Pitting of transfusion tracheids in cupressaceae. Aust J Bot 36(1):81-92

Gamalei Y (1989) Structure and function of leaf minor veins in trees and herbs. Trees 3:96-110

Gambles RL, Dengler RE (1982) The anatomy of the leaf of red pine, Pinus resinosa 2. Vasc tissues Can J Bot 60(12):28042824

Gil L (2010) Sucrose transporters play a role in phloem loading of CMV-infected melon plants that are defined as symplastic loaders. Presentation given at the International conference on Plant Vascular Biology 2010. Columbus, Ohio

Glockmann C, Kollmann R (1996) Structure and development of cell connections in the phloem of Metasequoia glyptostroboides needles. 1. Ultrastructural aspects of modified primary plasmodesmata in Strasburger cells. Protoplasma 193(14):191-203

Glockmann C, Schulz A (1999) Phloem loading and transport in gymnosperms: an enigma. In: International conference on assimilate transport and partitioning, Newcastle, Australia, August 15-20 1999. Apstract 7-07, p. 99.

Harris WM (1972) Ultrastructural observations on Pinaceae leaf phloem. 1. Spring condition. New Phytol 71(1):169-173

Heimerdinger G (1951) Zur Mikrotopographie der Saftströme im Transfusionsgewebe der Koniferennadel. 2. Entwicklungsgeschichte und Physiologie. Planta 40(2):93-111

Itaya A, Woo YM, Masuta C, Bao YM, Nelson RS, Ding B (1998) Developmental regulation of intercellular protein trafficking through plasmodesmata in tobacco leaf epidermis. Plant Physiol 118(2):373-385

Kaku S (1971) Possible role of endodermis as a barrier for ice propagation in freezing of pine needles. Plant Cell Physiol 12 (6):941-948 
Kim I, Zambryski PC (2005) Cell-to-cell communication via plasmodesmata during Arabidopsis embryogenesis. Curr Opin Plant Biol 8(6):593-599. doi:10.1016/j.pbi.2005.09.013

Kühn C, Quick WP, Schulz A, Riesmeier JW, Sonnewald U, Frommer WB (1996) Companion cell-specific inhibition of the potato sucrose transporter SUT1. Plant Cell Environ 19(10):1115-1123

Lazzaro MD, Thomson WW (1996) The vacuolar-tubular continuum in living trichomes of chickpea (Cicer arietinum) provides a rapid means of solute delivery from base to tip. Protoplasma 193 $(1-4): 181-190$

Lederer B (1955) Vergleichende Untersuchungen über das Transfusionsgewebe einiger rezenter Gymnospermen. In: Huber B (ed) Vergleichend-anatomische Untersuchungen. Gustav Fischer, Jena, pp 1-42

Lucas WJ, Ham B-K, Kim J-Y (2009) Plasmodesmata—bridging the gap between neighboring plant cells. Trends Cell Biol 19:495-500

Münch E (1930) Die Stoffbewegungen in der Pflanze. Gustav Fischer, Jena

Pallas JE, Mollenhauer HH (1972) Electron-microscopic evidence for plasmodesmata in dicotyledonous guard cells. Science 175 (4027):1275-1276

Parameswaran N, Liese W (1970) Cytology of Strasburger cells in conifer needles. Naturwissenschaften 57(1):45-46

Pina A, Errea P, Schulz A, Martens HJ (2009) Cell-to-cell transport through plasmodesmata in tree callus cultures. Tree Physiol 29 (6):809-818. doi:10.1093/treephys/tpp025

Rennie EA, Turgeon R (2009) A comprehensive picture of phloem loading strategies. P Natl Acad Sci USA 106(33):14162-14167. doi:10.1073/pnas.0902279106

Roden JS, Canny MJ, Huang CX, Ball MC (2009) Frost tolerance and ice formation in Pinus radiata needles: ice management by the endodermis and transfusion tissues. Funct Plant Biol 36(2):180 189. doi:10.1071/Fp08247

Sack L, Holbrook NM (2006) Leaf hydraulics. Annu Rev Plant Biol $57: 361-381$

Scholz F, Bauch J (1973) Anatomische und physiologische Untersuchungen zur Wasserbewegung in Kiefernnadeln. Planta 109 (2):105-119. doi:10.1007/bf00386118

Schulz A (1990) Conifers. In: Behnke H-D, Sjolund RD (eds) Comparative structure, induction and development. Springer, Berlin Heidelberg New York, pp 63-88

Schulz A (1992) Living sieve cells of conifers as visualized by confocal, laser-scanning fluorescence microscopy. Protoplasma 166(3-4):153-164

Schulz A (1995) Plasmodesmal widening accompanies the short-term increase in symplasmic phloem unloading in pea root-tips under osmotic-stress. Protoplasma 188(1-2):22-37

Schulz A (1999) Physiological control of plasmodesmal gating. In: van Bel AJE (ed) Plasmodesmata - stucture, function, role in cell communication. Springer, New York, pp 173-204

Schulz A (2005) Role of plasmodesmata in solute loading and unloading. In: Oparka K (ed) Plasmodesmata. Blackwell, Oxford, pp 135-161

Shiroya T, Lister GR, Slankis V, Krotkov G, Nelson CD (1962) Translocation of the products of photosynthesis to roots of pine seedlings. Can J Bot 40:1125-1135
Soar I (1922) The structure and function of the endodermis in the leaves of the abietineae. New Phytol 21(5):269-292. doi:10.1111/ j.1469-8137.1922.tb07604.x

Soda C, Bussotti F, Grossoni P, Barnes J, Mori B, Tani C (2000) Impacts of urban levels of ozone on Pinus halepensis foliage. Environ Exp Bot 44(1):69-82

Strasburger E (1891) Ueber den Bau und die Verrichtungen der Leitungsbahnen in den Pflanzen. Gustav Fischer, Jena

Turgeon R (2010) The role of phloem loading reconsidered. Plant Physiol 152(4):1817-1823. doi:10.1104/pp.110.153023

Turgeon R, Medville R (1998) The absence of phloem loading in willow leaves. Proc Natl Acad Sci USA 95:12055-12060

Turgeon R, Medville R (2010) Amborella trichopoda, plasmodesmata, and the evolution of phloem loading. Protoplasma

Turgeon R, Medville R, Nixon KC (2001) The evolution of minor vein phloem and phloem loading. Am J Bot 88(8):1331-1339

Voicu MC, Cooke JEK, Zwiazek JZ (2009) Aquaporin gene expression and apoplastic water flow in bur oak (Quercus macrocarpa) leaves in relation to the light response of leaf hydraulic conductance. J Exp Bot 60(14):4063-4075. doi:10.1093/jxb/erp239

Walles B (1973) On the ultrastructure of needles of Pinus silvestris. 1. vol 106. Studia forestalia, suecica. Allmänna Förlaget, Stockholm

Wille AC, Lucas WJ (1984) Ultrastructural and histochemical studies on guard-cells. Planta 160(2):129-142

Willenbrink J (2002) Assimilate transport in phloem: regulation and mechanism. Russ J Plant Physiol 49:8-15. doi:10.1007/ bf00225352

Willenbrink J, Kollmann R (1966) Über den Assimilattransport im Phloem von Metasequoia. Z Pflanzenphysiol 55:42-53

Worsdell WC (1897) Transfusion-tissue: its origin and function in the leaves of gymnospermous plants. Botany 5:301-319

Wu XQ, Zhu JM, Huang RZ, Wang QL, Zheng WJ, Hu YX, Lin JX (2001) Evidence of Casparian strip in the foliar endodermis of Pinus bungeana. Acta Bot Sin 43(10):1081-1084

Wu XQ, Lin JX, Zhu JM, Hu YX, Hartmann K, Schreiber L (2003) Casparian strips in needles of Pinus bungeana: isolation and chemical characterization. Physiol Plant 117 (3):421-424

Wu XQ, Lin JX, Lin QQ, Wang J, Schreiber L (2005) Casparian strips in needles are more solute permeable than endodermal transport barriers in roots of Pinus bungeana. Plant Cell Physiol 46 (11):1799-1808. doi:10.1093/Pcp/Pci194

Zhang CK, Turgeon R (2009) Downregulating the sucrose transporter VPSUT1 in Verbascum phoeniceum does not inhibit phloem loading. P Natl Acad Sci USA 106(44):18849-18854. doi:10.1073/pnas.0904189106

Zwieniecki MA, Boyce CK, Holbrook NM (2004) Functional design space of single-veined leaves: role of tissue hydraulic properties in constraining leaf size and shape. Ann Bot Lond 94(4):507513. doi:10.1093/Aob/Mch173

Zwieniecki MA, Brodribb TJ, Holbrook NM (2007) Hydraulic design of leaves: insights from rehydration kinetics. Plant Cell Environ 30(8):910-921. doi:10.1111/j.1365-3040.2007.001681.x 